\title{
ON A UNILATERAL PROBLEM ASSOCIATED WITH ELLIPTIC OPERATORS ${ }^{1}$
}

\author{
PETER HESS
}

\begin{abstract}
Let $\mathscr{A}$ be a uniformly elliptic linear differential expression of second order, defined on the bounded domain $\Omega \subset \boldsymbol{R}^{m}$, and let $\beta \subset \boldsymbol{R} \times \boldsymbol{R}$ be a maximal monotone graph. Under some growth assumption on $\beta$ it is shown that for any given $f \in L^{2}(\Omega)$ the problem: $\mathscr{A} u+\beta(u) \ni f$ on $\Omega, u=0$ on $\partial \Omega$, admits a strong solution. It is not required that $\mathscr{A}$ is monotone.
\end{abstract}

1. Introduction. Let $\Omega \subset R^{m}(m \geqq 1)$ be an open bounded set with smooth boundary $\partial \Omega$. Let further

where

$$
\mathscr{A}=\sum_{|\alpha|,|\beta| \leqq 1}(-1)^{|\alpha|} D^{\alpha} a_{\alpha \beta} D^{\beta},
$$

$$
\begin{aligned}
a_{\alpha \beta} \in C^{|\alpha|}(\bar{\Omega}) \quad(|\alpha|,|\beta| \leqq 1), & \\
\sum_{|\alpha|=|\beta|=1} a_{\alpha \beta} \xi_{\alpha} \xi_{\beta} \geqq c|\xi|^{2}, & c>0, \quad x \in \Omega, \quad \forall \xi \in \boldsymbol{R}^{m},
\end{aligned}
$$

be a uniformly elliptic differential expression of second order (all our spaces are assumed to be real). Moreover let $\beta \subset \boldsymbol{R} \times \boldsymbol{R}$ be a maximal monotone graph, i.e. a mapping of $R$ to $2^{R}$ such that (i) $\forall t_{1} \in \beta\left(s_{1}\right)$, $t_{2} \in \beta\left(s_{2}\right)$, the inequality $\left(t_{1}-t_{2}\right)\left(s_{1}-s_{2}\right) \geqq 0$ holds, and (ii) there exists no monotone graph in $\boldsymbol{R} \times \boldsymbol{R}$ extending $\beta$ properly. Let $D(\beta)=\{s \in \boldsymbol{R}$ : $\beta(s) \neq \varnothing\}$. Then for each $s \in D(\beta), \beta(s)$ is a closed interval. Suppose $0 \in \beta(0)$.

We prove in the present paper that for any $f \in L^{2}(\Omega)$ the boundary value problem

$$
\begin{array}{rr}
\mathscr{A} u+\beta(u) \ni f & \text { on } \Omega, \\
u=0 & \text { on } \partial \Omega
\end{array}
$$

Received by the editors July 11, 1972 .

AMS (MOS) subject classifications (1970). Primary 35J60, 47H05; Secondary 46E35.

Key words and phrases. Uniformly elliptic linear differential operator, maximal monotone mapping, Yosida approximation, homotopy arguments, Sobolev space, strong solution.

${ }^{1}$ Research supported by the Schweizerischer Nationalfonds.

(c) American Mathematical Society 1973 
admits a strong solution $u$, provided

Here

$$
\left|\beta^{0}(s)\right| \geqq \psi(s)|s| \text { with } \lim _{|s| \rightarrow \infty} \psi(s)=+\infty .
$$

and $\beta^{0}(s) s \geqq 0(s \in D(\beta))$.

$$
\begin{aligned}
\left|\beta^{0}(s)\right| & =\underset{t \in \beta(s)}{\operatorname{Min}}|t|, & & s \in D(\beta), \\
& =+\infty, & & \text { otherwise, }
\end{aligned}
$$

EXAMPLE. Let

$$
\begin{aligned}
\beta(s) & =(-\infty, 0], & & s=0, \\
& =s e^{s}, & & s>0 .
\end{aligned}
$$

Then we solve the following problem:

$$
\begin{gathered}
u \geqq 0 \quad \text { on } \Omega, \quad u=0 \quad \text { on } \partial \Omega ; \\
u(x)>0: \mathscr{A} u+u e^{u}=f, \\
u(x)=0: \quad \mathscr{A} u \geqq f .
\end{gathered}
$$

If $\mathscr{A}$ consists only of its "principal" part $\mathscr{A}^{\prime}=\sum_{|\alpha|=|\beta|=1}(-1)^{|\alpha|} D^{\alpha} a_{\alpha \beta} D^{\beta}$, with possibly a term of order 0 with nonnegative coefficient added, the strong solvability of $(*)$ follows (without the additional assumption (1)) from the theory of maximal monotone operators (e.g. Brézis-CrandallPazy [3, Example 1]). We reduce our more general case to the monotone situation by employing a homotopy argument.

2. Statement of the result. Let $A: L^{2}(\Omega) \supset D(A) \rightarrow L^{2}(\Omega)$ be the linear operator given by

$$
D(A)=H_{0}^{1}(\Omega) \cap H^{2}(\Omega), \quad A u=\mathscr{A} u, \quad u \in D(A) .
$$

The maximal monotone graph $\beta \subset \boldsymbol{R} \times \boldsymbol{R}$ extends uniquely to a (maximal monotone) operator $\bar{\beta}: L^{2}(\Omega) \rightarrow 2^{L^{2}(\Omega)}$ by

$$
v \in \bar{\beta}(u) \Leftrightarrow v(x) \in \beta(u(x)) \text { a.e. on } \Omega \text {. }
$$

THEOREM. Let $A$ and $\bar{\beta}$ be the operators defined in (2) and (3), respectively, and suppose (1) holds. Then for any given $f \in L^{2}(\Omega)$ there exists $u \in L^{2}(\Omega)$ with $A u+\bar{\beta}(u) \ni f$; i.e., $u \in H_{0}^{1}(\Omega) \cap H^{2}(\Omega)$, and for some $b \in L^{2}(\Omega)$ we have

$$
\begin{array}{ll}
b(x) \in \beta(u(x)) & \text { a.e. on } \Omega, \\
(\mathscr{A} u)(x)+b(x)=f(x) & \text { a.e. on } \Omega .
\end{array}
$$

Remarks. 1. One can show that $A+\bar{\beta}: L^{2}(\Omega) \rightarrow 2^{L^{2}(\Omega)}$ is a generalized pseudo-monotone operator in the sense of Browder-Hess [5]. This 
mapping is in general however neither densely defined nor quasi-bounded, so that the abstract existence theorems of [5] do not apply.

2. It might seem simpler to study problem (*) with operators acting from $H_{0}^{1}(\Omega)$ to $2^{H^{-1}(\Omega)}$. But besides loosing regularity of the solutions, one encounters serious interpretational difficulties concerning the (maximal monotone) operator $\tilde{\beta}: H_{0}^{1}(\Omega) \rightarrow 2^{H^{-1}(\Omega)}$ derived from $\bar{\beta}$ in an obvious way.

3 . For the weak solvability of somewhat related problems see the author's notes [8]-[10].

3. A solvability criterion for functional equations. Let $X$ be a real reflexive Banach space. We denote by $X^{*}$ its conjugate space, and by $(f, u)$ the value of $f \in X^{*}$ at $u \in X$. A mapping $T$ from $X$ to $X^{*}$ is called of type $(S)^{+}$provided for any sequence $\left\{u_{n}\right\}$ in $X$ converging weakly to some $u$, for which $\lim \sup \left(T u_{n}, u_{n}-u\right) \leqq 0$, the strong convergence $u_{n} \rightarrow u$ follows (" $\rightarrow$ " and " $\rightarrow$ " denote strong and weak convergence, respectively). The operator $T$ is further demicontinuous if $v_{n} \rightarrow v$ implies $T v_{n} \rightarrow T v$. For a subset $G$ of $X, \operatorname{cl}(G)$ means its closure, $\partial G$ its boundary.

Proposition. Let $X$ be a real reflexive Banach space, $G$ an open bounded subset of $X$ containing 0 , and $T_{t} u=T(u, t)$ a mapping of $X \times[0,1]$ into $X^{*}$ having the following properties:

(i) For fixed $t, T_{t}$ is demicontinuous and of type $(S)^{+}$;

(ii) $T_{t} u$ is continuous in $t$, uniformly with respect to $u \in \operatorname{cl}(G)$;

(iii) $\left(T_{0} u, u\right) \geqq 0, \forall u \in \partial G$.

Suppose that $T_{t} u \neq 0, \forall u \in \partial G, \forall 0 \leqq t \leqq 1$. Then the equation $T_{1} u=0$ is solvable in $G$.

The proof resembles that of Theorem 1 in Hess [7]; we do not reproduce it here.

4. Some remarks on maximal monotone operators. Let $\beta \subset \boldsymbol{R} \times \boldsymbol{R}$ be a maximal monotone graph with $0 \in \beta(0)$. Then the mapping $I+\lambda \beta$ is surjective for each $\lambda>0$, and $(I+\lambda \beta)^{-1}$ is a contraction in $\boldsymbol{R}$. The Yosida approximation $\beta_{\lambda}(\lambda>0)$ of $\beta$ is defined by $\beta_{\lambda}(s)=\lambda^{-1}\left(I-(I+\lambda \beta)^{-1}\right)(s)$; it is a monotone increasing, Lipschitz continuous function defined on $\boldsymbol{R}$. Clearly $0=\beta_{\lambda}(0)$ for each $\lambda>0$.

Let $\bar{\beta}: L^{2}(\Omega) \rightarrow 2_{L^{2}(\Omega)}$ and $\bar{\beta}_{\lambda}: L^{2}(\Omega) \rightarrow L^{2}(\Omega)$ denote the extensions of $\beta$ and $\beta_{\lambda}$, respectively, as defined in (3). One proves easily that $\bar{\beta}$ and $\bar{\beta}_{\lambda}$ are maxima monotone mappings, with $\overline{\beta_{\lambda}}$ being Lipschitz continuous. Moreover $\overline{\beta_{\lambda}}$ is the Yosida approximation of $\bar{\beta}: \overline{\beta_{\lambda}}=(\bar{\beta})_{\lambda}$. Since $0=\bar{\beta}_{\lambda}(0)$ for $\lambda>0$, we have $\left(\overline{\beta_{\lambda}}(u), u\right)_{L^{2}(\Omega)} \geqq 0$ for all $u \in L^{2}(\Omega)$.

For an extensive treatment of maximal monotone operators in reflexive Banach spaces we refer to Browder [4]. Yosida approximations to $m$ accretive operators are discussed in Kato [11] (cf. also Crandall-Pazy [6]), 
to maximal monotone mappings in Brézis-Crandall-Pazy [3] and BrowderHess [5].

5. Proof of the Theorem. We set $H=L^{2}(\Omega)$ and $X=H_{0}^{1}(\Omega)$. By identification of the Hilbert space $H$ with its conjugate space, $X \subset H \subset X^{*}$. Let $(\cdot, \cdot)$ denote either the inner product in $H$, or the duality pairing between $X^{*}$ and $X$. We distinguish the norms in the various spaces by subscript. Let $\mathscr{A}^{\prime}=-\sum_{|\alpha|=|\beta|=1} D^{\alpha} a_{\alpha \beta} D^{\beta}$ be the "principal" part of $\mathscr{A}$ and $A^{\prime}$ the operator in $H$ induced by $\mathscr{A}^{\prime}$ on the domain $D\left(A^{\prime}\right)=X \cap H^{2}(\Omega)$. Then

$$
\left\|A^{\prime} u\right\|_{H} \geqq c_{1}\|u\|_{H^{2}(\Omega)}, \quad c_{1}>0, \quad \forall u \in D\left(A^{\prime}\right)
$$

(e.g. Agmon [1, Theorem 9.8]). Let further $\mathscr{A}^{\prime \prime}=\mathscr{A}-\mathscr{A}^{\prime}$ and $A^{\prime \prime}$ be the mapping in $H$ induced by $\mathscr{A}^{\prime \prime}$, with $D\left(A^{\prime \prime}\right)=X$.

(i) Let $f \in H$ be given. We claim that there exists $R>0$ such that

$$
\begin{aligned}
0 \notin A^{\prime} u+\bar{\beta}(u)+t\left(A^{\prime \prime} u-f\right), \\
\forall u \in D\left(A^{\prime}\right) \cap D(\bar{\beta}) \quad \text { with }\|u\|_{X}=R, \quad \forall 0 \leqq t \leqq 1 .
\end{aligned}
$$

Suppose to the contrary that to each $n$ we find $u_{n} \in D\left(A^{\prime}\right) \cap D(\bar{\beta})$ with $\left\|u_{n}\right\|_{X}=n$ and $0 \leqq t_{n} \leqq 1$ such that $0 \in A^{\prime} u_{n}+\bar{\beta}\left(u_{n}\right)+t_{n}\left(A^{\prime \prime} u_{n}-f\right)$. Let $b_{n} \in \bar{\beta}\left(u_{n}\right)$ be the element with

$$
0=A^{\prime} u_{n}+b_{n}+t_{n}\left(A^{\prime \prime} u_{n}-f\right) .
$$

Taking the inner product of (6) with $b_{n}$, we obtain

$$
\left(A^{\prime} u_{n}, b_{n}\right)+\left\|b_{n}\right\|_{H}^{2}=-t_{n}\left(A^{\prime \prime} u_{n}-f, b_{n}\right) .
$$

It is shown in Brézis [2, Lemma 2], that

$$
\left(A^{\prime} u_{n}, b_{n}\right) \geqq 0 .
$$

Let $v_{n}=n^{-1} u_{n}$. Dividing (7) by $n^{2}$ and observing (8) we get

$$
\left\|n^{-1} b_{n}\right\|_{H}^{2} \leqq-t_{n}\left(A^{\prime \prime} v_{n}, n^{-1} b_{n}\right)+t_{n} n^{-1}\left(f, n^{-1} b_{n}\right) .
$$

This implies that the sequence $\left\{n^{-1} b_{n}\right\}$ is bounded in $H$. Thus also $A^{\prime} v_{n}$ remains bounded in $H$ as $n \rightarrow \infty$. By (4) and Rellich's compactness theorem we may pass to a subsequence and assure that $v_{n} \rightarrow v$ in $X$ as well as a.e. on $\Omega$. Hence $v \neq 0$.

On the other hand, by (1),

$$
\int_{\Omega} \psi\left(u_{n}\right) u_{n}^{2} d x \leqq\left(b_{n}, u_{n}\right) \leqq-t_{n}\left(A^{\prime \prime} u_{n}-f, u_{n}\right) .
$$


It follows that

consequently

$$
\int_{\Omega} \psi\left(u_{n}\right) v_{n}^{2} d x \leqq\left|\left(A^{\prime \prime} v_{n}-n^{-1} f, v_{n}\right)\right|
$$

$$
\lim \sup \int_{\Omega} \psi\left(u_{n}(x)\right) v_{n}^{2}(x) d x \leqq\left|\left(A^{\prime \prime} v, v\right)\right| .
$$

But $\psi\left(u_{n}(x)\right)=\psi\left(n v_{n}(x)\right) \rightarrow+\infty$ where $v(x) \neq 0$. Hence $v(x)=0$ a.e., in contradiction to $v \neq 0$. We have proved that (5) holds for some $R>0$.

(ii) For $\lambda>0$ let $\bar{\beta}_{\lambda}$ be the Yosida approximation of $\bar{\beta}$ as described in $\S 4$. We assert the existence of a positive constant $\lambda_{0}$ having the property that

$$
0 \neq A^{\prime} u+\overline{\beta_{\lambda}}(u)+t\left(A^{\prime \prime} u-f\right),
$$

$$
\forall u \in D\left(A^{\prime}\right) \quad \text { with }\|u\|_{X}=R, \quad \forall 0 \leqq t \leqq 1, \quad \forall 0<\lambda<\lambda_{0} .
$$

Suppose to each $n$ there exist $u_{n} \in D\left(A^{\prime}\right),\left\|u_{n}\right\|_{X}=R, 0 \leqq t_{n} \leqq 1$, and $0<\lambda_{n}<n^{-1}$, such that

Then

$$
0=A^{\prime} u_{n}+\overline{\beta_{\lambda_{n}}}\left(u_{n}\right)+t_{n}\left(A^{\prime \prime} u_{n}-f\right) .
$$

$$
0=\left(A^{\prime} u_{n}, \overline{\beta_{\lambda_{n}}}\left(u_{n}\right)\right)+\left\|\overline{\beta_{\lambda_{n}}}\left(u_{n}\right)\right\|_{H}^{2}+t_{n}\left(A^{\prime \prime} u_{n}-f, \overline{\beta_{\lambda_{n}}}\left(u_{n}\right)\right) .
$$

Since $\left(A^{\prime} u_{n}, \overline{\beta_{\lambda_{n}}}\left(u_{n}\right)\right) \geqq 0$ and $t_{n}\left(A^{\prime \prime} u_{n}-f\right)$ remains bounded in $H$ as $n \rightarrow \infty$, we infer as above the boundedness of the sequences $\left\{\overline{\beta_{\lambda_{n}}}\left(u_{n}\right)\right\}$ and $\left\{A^{\prime} u_{n}\right\}$ in $H$. By (4) and the closedness of the mapping $A^{\prime}$ in $H$ we may assume (after passage to subsequences) that

$$
\begin{aligned}
u_{n} \rightarrow u_{0} & \text { in } X(\text { and thus in } H), \\
A^{\prime} u_{n} \rightarrow A^{\prime} u_{0} & \text { in } H,
\end{aligned}
$$

and

$$
\overline{\beta_{\lambda_{n}}}\left(u_{n}\right) \rightarrow z \text { in } H \text {. }
$$

Hence $u_{0} \in D\left(A^{\prime}\right)$ and $\left\|u_{0}\right\|_{X}=R$. By (10), (12) and a result on Yosida approximations (e.g. Kato [11, Lemma 4.5]), $u_{0} \in D(\bar{\beta})$ and $z \in \bar{\beta}\left(u_{0}\right)$. Assuming further that $t_{n} \rightarrow t_{0}$ in $[0,1]$, we infer that

contradicting (5).

$$
\begin{gathered}
0 \in A^{\prime} u_{0}+\bar{\beta}\left(u_{0}\right)+t_{0}\left(A^{\prime \prime} u_{0}-f\right), \\
u_{0} \in D\left(A^{\prime}\right) \cap D(\bar{\beta}), \quad\left\|u_{0}\right\|_{X}=R,
\end{gathered}
$$

(iii) Let $A_{0}^{\prime}: X \rightarrow X^{*}$ be the bounded linear operator given by

$$
\left(A_{0}^{\prime} u, v\right)=\sum_{|\alpha|=|\beta|=1} \int_{\Omega} a_{\alpha \beta} D^{\beta} u D^{\alpha} v d x \quad \forall u, v \text { in } X .
$$


It follows from (9) that

$$
\begin{gathered}
0 \neq A_{0}^{\prime} u+\overline{\beta_{\lambda}}(u)+t\left(A^{\prime \prime} u-f\right), \\
\forall u \in X \quad \text { with }\|u\|_{X}=R, \quad \forall 0 \leqq t \leqq 1, \quad \forall 0<\lambda<\lambda_{0} .
\end{gathered}
$$

Indeed, assume

$$
0=A_{0}^{\prime} u_{1}+\overline{\beta_{\lambda_{1}}}\left(u_{1}\right)+t_{1}\left(A^{\prime \prime} u_{1}-f\right)
$$

for some $u_{1} \in X$ with $\left\|u_{1}\right\|_{X}=R, 0 \leqq t_{1} \leqq 1$, and $0<\lambda_{1}<\lambda_{0}$. Then

$$
0=\left(A_{0}^{\prime} u_{1}, \varphi\right)+\left(\overline{\beta_{\lambda_{1}}}\left(u_{1}\right), \varphi\right)+t_{1}\left(A^{\prime \prime} u_{1}-f, \varphi\right)
$$

$\forall \varphi \in C_{0}^{\infty}(\Omega)$. Since $\overline{\beta_{\lambda_{1}}}\left(u_{1}\right)+t_{1}\left(A^{\prime \prime} u_{1}-f\right) \in H$ by the Lipschitz continuity of $\beta_{\lambda_{1}}$, it follows from regularity results on linear elliptic operators (e.g. Agmon [1, Theorem 9.8]), that $u_{1} \in H^{2}(\Omega)$. But for $u_{1} \in D\left(A^{\prime}\right), A_{0}^{\prime} u_{1}=$ $A^{\prime} u_{1}$. Hence (14) constitutes a violation of (9).

As an immediate consequence of the Gårding inequality

$$
\left(A_{0}^{\prime} u, u\right) \geqq c_{2}\|u\|_{X}^{2}, \quad c_{2}>0, \quad \forall u \in X,
$$

the Rellich theorem on compactness of the embedding $X \subset H$, and the monotonicity of $\overline{\beta_{\lambda}}$, the mapping

$$
A_{0}^{\prime}+\overline{\beta_{\lambda}}+t\left(A^{\prime \prime}-f\right): X \rightarrow X^{*}
$$

is of type $(S)^{+}$for each $0 \leqq t \leqq 1$ and $\lambda>0$. By (13) and the Proposition, the equation $0=A_{0}^{\prime} u_{\lambda}+\overline{\beta_{\lambda}}\left(u_{\lambda}\right)+A^{\prime \prime} u_{\lambda}-f$ is solvable for $0<\lambda<\lambda_{0}$, with $\left\|u_{\lambda}\right\|_{X}<R$. We conclude as above that $u_{\lambda} \in D\left(A^{\prime}\right)$; thus

$$
A u_{\lambda}+\overline{\beta_{\lambda}}\left(u_{\lambda}\right)=f \text {. }
$$

(iv) In a similar way as in (ii) we now pass to the limit $\lambda \downarrow 0$ in (15) and obtain a desired solution of $A u+\bar{\beta}(u) \ni f$. Q.E.D.

\section{REFERENCES}

1. S. Agmon, Lectures on elliptic boundary value problems, Van Nostrand Math. Studies, no 2, Van Nostrand, Princeton, N.J., 1965. MR 31 \#2504.

2. H. Brézis, Nouveaux théorèmes de régularité pour les problèmes unilatéraux (to appear).

3. H. Brézis, M. G. Crandall and A. Pazy, Perturbations of nonlinear maximal monotone sets in Banach space, Comm. Pure Appl. M Iath. 23 (1970), 123-144.

4. F. E. Browder, Nonlinear operators and nonlinear equations of evolution in Banach spaces, Proc. Sympos. Pure Math., vol. 18, part 2, Amer. Math. Soc., Providence, R.I. (to appear).

5. F. E. Browder and P. Hess, Nonlinear mappings of monotone type in Banach spaces, J. Functional Analysis 11 (1972), 251-294. 
6. M. G. Crandall and A. Pazy, Semi-groups of nonlinear contractions and dissipative sets, J. Functional Analysis 3 (1969), 376-418. MR 39 \#4705.

7. P. Hess, On nonlinear mappings of monotone type homotopic to odd operators, J. Functional Analysis 11 (1972), 138-167.

8. - On nonlinear mappings of monotone type with respect to two Banach spaces, J. Math. Pures Appl. 52 (1973), 13-26.

9. —_ Variational inequalities for strongly nonlinear elliptic operators, J. Math. Pures Appl. (to appear).

10. $\longrightarrow$, A strongly nonlinear elliptic boundary value problem, J. Math. Anal. Appl. (to appear).

11. T. Kato, Accretive operators and nonlinear evolution equations in Banach spaces Proc. Sympos. Pure Math., vol. 18, part 1, Amer. Math. Soc., Providence, R.I., 1970, pp. 138-161. MR 42 \#6663.

Mathematics Institute, University OF Zürich, ZÜRICH, Switzerland 